\title{
Predictors of Chronic Abdominal Pain Affecting the Well-Being of Children in Primary Care
}

Leo A.A. Spee, $M D^{1}$

Yvonne Lisman-van Leeuwen, $\mathrm{PbD}^{1,2}$

Marc A. Benninga, MD, $P b D^{3}$

Sita M. A. Bierma-Zeinstra, $P b D^{1}$

Boudewijn J. Kollen, $P b D^{2}$

Marjolein Y. Berger, $M D, P b D^{1,2}$

'Department of General Practice, Erasmus MC University Medical Center, Rotterdam, the Netherlands

${ }^{2}$ Department of General Practice, University of Groningen, University Medical Center Groningen, Groningen, the Netherlands

${ }^{3}$ Department of Pediatric Gastroenterology, Emma Children's Hospital/Academic Medical Center, Amsterdam, the Netherlands

Conflicts of interest: authors report none.

\section{CORRESPONDING AUTHOR}

Leo A. A. Spee, MD

Department of General Practice, NA-1924

Erasmus MC University Medical Centre

PO Box 2040, 3000 CA

Rotterdam, the Netherlands

1.spee@erasmusmc.nl

\begin{abstract}
PURPOSE Abdominal pain is a frequent symptom among children but is rarely associated with organic disease. Although it may persist for years, no factors have been identified that predict its prognosis. Our aim was to determine whether patient characteristics at initial consultation can predict chronic abdominal pain severe enough to influence the child's well-being at 1 year of follow-up.
\end{abstract}

METHODS We conducted this prospective cohort study in primary care, including consecutive children aged 4 to 17 years seen for abdominal pain by their family physician. Multivariate logistic regression analysis was used to identify prognostic factors that predicted chronic abdominal pain 1 year later. Discriminative ability of identified predictors was assessed using the area under the receiver operating characteristic curve and explained variance.

RESULTS The risk of having chronic abdominal pain at 1 year of follow-up was $37.1 \%$ in the cohort overall. Increasing age, waking up at night with pain, high levels of other somatic complaints, and chronic abdominal pain at baseline independently predicted chronic abdominal pain at 1 year. These predictors had a poor to moderate discriminative ability, however; the area under the receiver operating characteristic curve was only 0.69 , and the predictors collectively explained only $14.3 \%$ of variance in the development of chronic abdominal pain. The absolute risk ranged from $19.4 \%$ among children having none of the predictors to $65.5 \%$ among children having 3 or 4 predictors.

CONCLUSIONS Chronic abdominal pain sufficient to affect well-being is common among children initially seen for abdominal pain by family physicians. Although the risk of this outcome increases with number of predictors, these predictors are of limited value in identifying children in whom pain will become chronic, suggesting that other, as yet unidentified factors play an important role.

Ann Fam Med 2015;13:158-163. doi: 10.1370/afm.1736.

\section{INTRODUCTION}

A bdominal pain in children accounts for about $5 \%$ of childhood consultations in general practice in the Netherlands, ${ }^{1}$ which is in agreement with studies performed in Australia and the United States reporting similar numbers of $2 \%$ to $4 \%{ }^{2,3}$ Most cases are considered medically unexplained and are labeled as functional abdominal pain (FAP). ${ }^{4}$ In $10 \%$ to $15 \%$ of schoolchildren, abdominal pain persists or returns regularly, which increases health care use and has implications for the child's well-being in that they have poorer school attendance, withdraw from social activities, and show impaired physical abilities. ${ }^{5.7}$ One in 3 children with chronic FAP continues to experience this pain for at least 5 years, underscoring the burden of disease. ${ }^{8}$ In addition, childhood FAP has been linked to functional gastrointestinal (GI) disorders in adulthood, such as irritable bowel syndrome. . $^{913}$

Despite evidence that childhood FAP may persist for years, few factors have been identified that predict whether it interferes with the child's wellbeing. ${ }^{13}$ Recently, a high level of non-GI symptoms was found to be associated with a greater likelihood of functional GI disorders in young adulthood. ${ }^{14}$ This finding suggests that having other somatic symptoms, in addition to abdominal pain, may be a potentially useful clinical marker of increased risk 
for poor long-term outcomes in children with FAP. Having a parent with GI symptoms is another factor related to persistence of abdominal pain. ${ }^{12}$ Because these factors can easily be incorporated into the medical evaluation, they have potential clinical utility. Ideally, family physicians would be able to identify children at risk for long-term abdominal pain and make more appropriate management decisions at an earlier stage, such as using more stringent follow-up or consulting other (mental) health care workers. The present study therefore aimed to determine which patient characteristics easily incorporated into the initial medical evaluation could predict chronic abdominal pain (CAP) that is severe enough to interfere with a child's well-being 1 year later.

\section{METHODS}

\section{Design, Setting, and Patients}

This was an observational prospective cohort study with a 1-year follow-up. A total of 53 family physicians were recruited in Rotterdam, a large multicultural city, and its rural surroundings. The included practices comprise a population of about 16,000 children aged 4 to 17 years. During a 2-year period, consecutive children in this age-group consulting with a new symptom of abdominal pain were invited to participate in the study. Abdominal pain was defined as new if the child had not visited the family physician during the past 3 months for the same symptom. We excluded children with a previous diagnosis of inflammatory bowel disease, celiac disease, or lactose intolerance, and children unable to complete questionnaires because of language or cognitive problems. As this was an observational study, no diagnostic work-up tool was used, and the extent of diagnostic work-up to exclude possible organic disease was left to the family physician's discretion. Parental consent was obtained for all participants; child consent was also obtained for children aged 12 years or older. The study protocol was approved by the local ethics committee.

To check for possible selection bias, we compared important characteristics of eligible included children and eligible nonincluded children. Relative to eligible nonincluded children, the included children were younger (mean age $=8.5$ and 9.2 years, respectively), less frequently received a diagnosis of gastroenteritis, and more frequently received a diagnosis of generalized abdominal pain; no difference in sex was found. The design of the study and representativeness of included children are described in detail elsewhere. ${ }^{15}$

\section{Outcome Definition}

The primary outcome measure was CAP that interfered with the child's well-being at 1 year of follow-up. This outcome was defined as the presence of abdomi- nal pain at least once each month in the past 3 months, severe enough for the child to stay home from school, to stop or avoid play, to take medication for the pain, or to rate the pain as moderate to severe. ${ }^{16}$

\section{Potential Predictors}

For potential positive predictors of CAP, we chose to investigate factors that could easily be incorporated into the initial medical evaluation. First, we selected candidate predictors on the basis of the results of a systematic review of predictors of childhood CAP in specialist care $^{13}$ - age, sex, and a family history of GI symptomsand we investigated dyspepsia due to abdominal pain as it is thought to be positively associated with CAP. We also investigated levels of other somatic symptoms in children, in addition to the abdominal pain, because of their reported association with poor long-term outcome, $^{12}$ and body mass index because of a possible association between overweight and recurrent abdominal pain. ${ }^{17}$ We investigated waking up at night because of abdominal pain as a possible positive predictor. Historically, this factor has been considered a red flag symptom, but more recent data suggest an association with FAP. ${ }^{18,19}$

In addition to assessing positive predictors after 1 year, we also investigated several red flag symptoms: vomiting, fever, blood on stools, and dysuria. We hypothesized that these symptoms are related to organic diseases that are mostly self-limiting or worsen in the long term, thus being recognized and treated as such. We therefore label these red flag symptoms as negative predictors, that is, we expected them to predict the absence of CAP. Furthermore, we added epigastric pain as a red flag symptom as a systematic review found a significant association between such pain and Helicobacter pylori infection in children. ${ }^{20}$

\section{Measurements}

Data on age, sex, red flag symptoms, and family history were obtained using self-administered standardized questionnaires (completed by children if they were aged 9 years or older, by parents, or by both). Children were considered to have a positive family history of GI symptoms when at least 1 parent reported a history of irritable bowel syndrome or $H$ pylori gastritis, or if 1 parent, or a first- or second-degree relative, had a history of inflammatory bowel disease or CAP. Dyspepsia was defined as belching, bloating, regurgitation, or a combination thereof. Data on the child's defecation were obtained using a standardized bowel questionnaire. The child's body mass index was categorized as normal or overweight using child-adjusted cutoff points. ${ }^{21}$ Pain intensity was determined on an 11-point numerical rating scale in children aged 8 to 17 years and on a 6 -point pain faces scale for children 
younger than 8 years. ${ }^{22}$ Moderate to severe pain was defined as a score of 3 or higher on the numerical rating scale and 4 or higher on the pain faces scale. CAP at baseline was assessed and defined as described above. For assessment of other somatic symptoms, we used the somatic syndrome scale of the Child Behavior Checklist, ${ }^{23,24}$ which identifies children with medically unexplained (somatic) symptoms at a level comparable to that in referred children with somatization problems (Table 1). These children were defined as having high levels of other somatic symptoms.

\section{Statistical Analyses}

Continuous variables are expressed as mean (SD) values. Binary and categorical variables are presented as counts and percentages of the number of patients responding per item. Differences between children included in the analyses and those lost to follow-up were analyzed using independent sample $t$ tests (age) and $\chi^{2}$ tests (sex and presence of CAP). Multiple imputation ( $\mathrm{n}=10$ data sets) of missing values was undertaken using an imputation model containing all predictor variables and the outcome measure.

Univariate logistic regression analyses were used to study the association between potential predictors and the primary outcome. For the multivariate prediction model, we performed a backward elimination approach with all the preselected candidate variables; at each step, the least significant variable was removed stepwise from this model. The final model contains only those variables that explained the outcome in our population at a significance level of $P<.157$, according to the Akaike information criterion. ${ }^{24}$ We determined odds ratios (ORs) for each variable, as well as the area under the receiver operating characteristic curve (ROC) for the model with 95\% CIs. The performance of the final prediction model was tested for explained variance (the Nagelkerke $R^{2}$ ), which shows

\section{Table 1. Overview of Questions on the Somatic Complaints Syndrome Scale of the Child Behavior Checklist}

\begin{tabular}{|c|c|}
\hline Questions for Children Aged 4-5 Years & Questions for Children Aged 6-16 Years \\
\hline Do you/does your child experience: & Do you/does your child experience: \\
\hline $\begin{array}{l}\text { Pain in general (without medical explana- } \\
\text { tion; not abdominal pain or headaches)? }\end{array}$ & $\begin{array}{l}\text { Pain in general (without medical explanation; } \\
\text { not abdominal pain or headaches)? }\end{array}$ \\
\hline Headaches? & Headaches? \\
\hline Nausea, feels sick? & Nausea, feels sick? \\
\hline Constipation? & Constipation? \\
\hline Vomiting, throwing up? & Vomiting, throwing up? \\
\hline Can't stand having things out of place? & Problems with eyes (not if corrected by glasses)? \\
\hline Diarrhea or loose bowels (when not sick)? & Rashes or other skin problems? \\
\hline Doesn't eat well? & Nightmares? \\
\hline Painful bowel movements? & Feels dizzy or lightheaded? \\
\hline Too concerned with neatness or cleanliness? & Overtired without good reason? \\
\hline
\end{tabular}

the percentage of variance between patients in the outcome that is explained by the predictors. Finally, we examined the odds of CAP by the number of identified predictors that children were exposed to, dichotomizing age as younger than 10 years, or 10 years or older. All analyses were conducted with SPSS for Windows, release 20.0 (SPSS, Inc).

\section{RESULTS}

\section{Study Sample}

A total of 348 children were recruited, of whom 305 (87.6\%) participated. After 1 year, 22 children (7.2\%) were lost to follow-up. One child was admitted to a hospital; the remaining 21 children were lost because they, their parents, or both were not interested in further participation ( 8 because of lack of time, 7 for unknown reasons, and 6 because they were free of abdominal pain). These children did not differ from the included children with respect to age, sex, and presence of CAP at baseline (data not shown).

Analyses were based on 283 children, of whom 177 $(62.5 \%)$ were girls; their mean age was 8.3 (SD 2.9) years. In total, 105 children $(37.1 \%)$ fulfilled the criteria for CAP at 1 year of follow-up. Univariate associations between patient characteristics and this outcome are shown in Table 2.

\section{Predictors of Pain}

In multivariate analysis, increasing age, waking up at night because of abdominal pain, high levels of other somatic symptoms, and CAP at baseline independently predicted the presence of CAP 1 year later (Table 3). The area under the ROC for the original data set was 0.69 (95\% CI, 0.63-0.76), and this model explained $14.3 \%$ of the variance in the outcome measure. After multiple imputation, the area under the $\mathrm{ROC}$ ranged from 0.66 to 0.69 , and the explained variance ranged from $9.8 \%$ to $13.6 \%$.

The number of positive predictors influenced the absolute risk of having CAP at 1 year. The risk ranged from $19.4 \%$ among children having none of the predictors to $65.5 \%$ among children having 3 or 4 predictors (Table 4 ).

Epigastric pain was the only negative candidate predictor that was associated with CAP at 1 year at our predefined 157 significance level $(P=.08)$. Adding epigastric pain to the multivariate model of positive predictors did not alter the area under the ROC, however. 
Table 2. Univariate Association of Candidate Predictors With Chronic Abdominal Pain

\begin{tabular}{|c|c|c|c|c|c|}
\hline Predictor & $\begin{array}{c}\text { CAP } \\
(n=105)\end{array}$ & $\begin{array}{l}\text { No CAP } \\
(n=178)\end{array}$ & $\begin{array}{c}\text { Original OR } \\
(95 \% \mathrm{Cl})^{\mathrm{a}}\end{array}$ & $\begin{array}{c}\text { Pooled OR } \\
(95 \% \mathrm{Cl})^{\mathrm{b}}\end{array}$ & $P$ Value ${ }^{c}$ \\
\hline \multicolumn{6}{|l|}{ Candidate positive predictors } \\
\hline Age, mean (SD), y & $8.8(3.2)$ & $8.0(2.7)$ & $1.10(1.02-1.20)$ & $1.09(1.00-1.18)$ & .05 \\
\hline Sex, female, No. (\%) & $73(69.5)$ & $104(58.4)$ & $1.62(0.97-2.71)$ & $1.47(0.89-2.44)$ & .14 \\
\hline Dyspepsia, No. (\%) & $53(50.5)$ & $74(44.0)$ & $1.30(0.79-2.11)$ & $1.27(0.78-2.06)$ & .33 \\
\hline Waking up at night because of pain, No. (\%) & $53(50.5)$ & $56(31.5)$ & $2.22(1.35-3.65)$ & $2.08(1.29-3.36)$ & .003 \\
\hline Overweight, No. $(\%)^{d}$ & $15(14.3)$ & $13(7.3)$ & $2.12(0.96-4.64)$ & $1.81(0.82-4.00)$ & .14 \\
\hline High level of other somatic symptoms, No. (\%) & $28(26.7)$ & $20(11.2)$ & $2.87(1.52-5.42)$ & $2.58(1.38-4.82)$ & .003 \\
\hline CAP at baseline, No. (\%) & $58(55.2)$ & $73(41.0)$ & $1.78(1.09-2.89)$ & $1.63(1.01-2.62)$ & .04 \\
\hline Parent with functional Gl complaints, No. (\%) & $37(35.6)$ & $49(28.0)$ & $1.42(0.85-2.39)$ & $1.60(0.88-2.90)$ & .13 \\
\hline \multicolumn{6}{|l|}{ Candidate negative predictors } \\
\hline Vomiting, No. (\%) & $16(15.2)$ & $30(16.9)$ & $0.89(0.46-1.72)$ & $0.91(0.47-1.75)$ & .77 \\
\hline Fever, No. (\%) & $20(19.0)$ & $28(15.7)$ & $1.26(0.67-2.37)$ & $1.23(0.65-2.31)$ & .52 \\
\hline Dysuria, No. (\%) & $8(7.6)$ & $9(5.1)$ & $1.55(0.58-4.15)$ & $1.36(0.31-3.62)$ & .53 \\
\hline Blood on stools, No. (\%) & $3(2.9)$ & $4(2.3)$ & $1.27(0.28-5.80)$ & $1.15(0.25-5.29)$ & .86 \\
\hline Epigastric pain, No. (\%) & $5(4.9)$ & $20(12.6)$ & $0.36(0.13-0.99)$ & $0.45(0.18-1.10)$ & .08 \\
\hline \multicolumn{6}{|c|}{$\mathrm{CAP}=$ chronic abdominal pain; $\mathrm{Gl}=$ gastrointestinal; $\mathrm{OR}=$ odds ratio. } \\
\hline \multicolumn{6}{|c|}{$\begin{array}{l}\text { a Original data set ORs. } \\
\text { b Pooled ORs after multiple imputation. } \\
\text { c } P \text { values of imputed data. } \\
{ }^{d} \text { According to body mass index using child-adjusted cutoff points. }{ }^{21}\end{array}$} \\
\hline
\end{tabular}

\section{DISCUSSION}

In the present study, 37.1\% of children had CAP 1 year after initial consultation for abdominal pain. We identified several positive predictors of CAP: increasing age, waking up at night due to abdominal pain, high levels of other somatic symptoms, and CAP at baseline. Epigastric pain was the only negative predictor identified.

To our knowledge, this is the first prospective cohort study in primary care to investigate potential predictors of poor outcome in children seen for abdominal pain. Furthermore, this is the first study to incorporate the impact on the child's well-being into the outcome measure. Ideally, family physicians would be able to identify children who continue to experi-

\section{Table 3. Multivariate Association of Candidate Predictors With Chronic Abdominal Pain}

\begin{tabular}{|c|c|c|}
\hline Predictor & OR $(95 \% \mathrm{Cl})^{a}$ & $P$ Value $^{b}$ \\
\hline \multicolumn{3}{|l|}{ Positive predictors } \\
\hline Age & $1.08(0.99-1.17)$ & .09 \\
\hline $\begin{array}{l}\text { Waking up at night because } \\
\text { of pain }\end{array}$ & $2.28(1.38-3.77)$ & .001 \\
\hline $\begin{array}{l}\text { High levels of other somatic } \\
\text { symptoms }\end{array}$ & $2.18(1.14-4.19)$ & .02 \\
\hline CAP at baseline & $1.77(1.07-2.95)$ & .03 \\
\hline \multicolumn{3}{|l|}{ Negative predictors } \\
\hline Epigastric pain & $0.45(0.18-1.10)$ & .08 \\
\hline
\end{tabular}

ence difficulties in daily functioning due to abdominal pain as this would allow them to make more appropriate management decisions at an earlier stage.

Our finding that $37.1 \%$ of children have CAP 1 year after initial consultation is in line with a systematic review that reported persistence of abdominal pain in $29.1 \%$ of children after a median follow-up of 5 years. ${ }^{7}$ Both our study and this review identified CAP at baseline to be a predictor for the persistence of abdominal pain, while another study among school-aged children in an open population identified a history of pain as a predictor for chronic benign pain in general. ${ }^{26}$

We found that older children were more likely to have CAP. In contrast, the aforementioned review reported conflicting evidence for an association between age and the persistence of chronic FAP. ${ }^{13} \mathrm{~A}$ more recent study of Helgeland et $\mathrm{al}_{1}{ }^{27}$ however, supports our finding. This latter study included children aged 8 to 15 years with recurrent abdominal pain referred to a general outpatient pediatric clinic; the authors reported a significant association between older age and higher levels of abdominal pain at 6 to 9 months of follow-up. A possible explanation is that parents with younger children may visit the family physician shortly after symptom onset, whereas they may be inclined to wait out the clinical course in older children; therefore, only a subset of older children with a longer duration of abdominal pain, with concurrent worse prognosis, might visit their family physician. Another explanation may be that reaching puberty is associated 


\begin{tabular}{|c|c|c|}
\hline $\begin{array}{l}\text { Number of } \\
\text { Predictors }^{\mathrm{a}}\end{array}$ & $\begin{array}{l}\text { Odds Ratio } \\
(95 \% \mathrm{Cl})\end{array}$ & $\begin{array}{l}\text { Absolute } \\
\text { Risk, }^{c} \%\end{array}$ \\
\hline 0 & Reference & 19.4 \\
\hline 1 & $2.25(0.92-5.48)$ & 34.9 \\
\hline 2 & $3.91(1.52-10.08)$ & 48.2 \\
\hline 3 or 4 & $8.00(2.90-22.09)$ & 65.5 \\
\hline \multicolumn{3}{|c|}{$\begin{array}{l}\text { a Out of age } \geq 10 \text { years, waking up at night because of pain, high levels of } \\
\text { other somatic symptoms, and chronic abdominal pain at baseline. }\end{array}$} \\
\hline \multicolumn{3}{|c|}{ b Pooled ORs after multiple imputation. } \\
\hline
\end{tabular}

with many psychological and physiologic changes that may cause abdominal pain to interfere with well-being.

Our finding that female sex is not a predictor of poor prognosis agrees with the existing strong evidence from the review of Gieteling et al. ${ }^{13}$ We could not support the reported finding of this review that having a parent with functional GI symptoms predicts a poor outcome of abdominal pain.

The association of the coexistence of multiple somatic complaints and childhood FAP is generally accepted. ${ }^{4}$ One study found that high levels of non-GI symptoms were associated with a greater likelihood of functional GI disorders in young adulthood. ${ }^{14}$ In agreement, we found a significant association between high levels of other somatic symptoms at baseline and CAP 1 year later, suggesting that such symptoms in children consulting for abdominal pain may help identify those at higher risk for poor prognosis.

Waking up at night because of abdominal pain predicted CAP. Although this symptom is a red flag symptom according to the pediatric Rome criteria III (PRC-III), none of the affected children had a severe organic illness. It is more likely to be an indicator of a sleeping disorder. The association between sleep disturbances and FAP has been reported: a primary care study from Australia found that children with abdominal pain had 4 times the risk of sleep disturbances..$^{18}$ Also, FAP is reportedly associated with increased difficulty initiating and maintaining sleep, symptoms that were defined as behavioral sleep-disorder symptoms, which are common in children with medical and psychiatric disorders. ${ }^{19}$

To our knowledge, we are the first to find a negative association between epigastric pain and CAP. A possible explanation is that epigastric pain is caused by, for example, gastritis or gastroenteritis, conditions that are mostly self-limiting. The hypothesis that epigastric pain is potentially caused by organic pathology is not new, however; a systematic review reported a possible association between epigastric pain in children and H pylori infection, and the Maastricht III consensus recommends testing of children with "upper gastrointestinal symptoms" for this bacterium. ${ }^{28}$ Another explanation may be that physicians tend to test more extensively in children with epigastric pain, which consequently results in a higher percentage being identified as having organic disease.

\section{Limitations}

This study had noteworthy limitations. First, we focused on potential predictors that could be incorporated in the medical evaluation during the first consultation for abdominal pain. Because long-term abdominal pain without organic cause is a complex interaction of biologic, psychological, and social factors, however, we may have missed some features of this symptom complex, such as essential psychosocial factors. This lack of capture of potentially relevant factors might account for the low explained variance of our model and only moderate ability to predict CAP.

Second, the follow-up period of 1 year was relatively short given that in about $29 \%$ of children with chronic FAP, the pain persists for approximately 5 years. ${ }^{8}$ An earlier study, however, identified 3 trajectories of childhood abdominal pain; a high-risk group did not improve at all over 5 years, whereas the low- and short-term risk groups showed improvement after only 2 months and maintained this improvement after 5 years. ${ }^{29}$ Our follow-up of 1 year is therefore probably justified and is representative for a poor prognosis.

\section{Implications}

We identified 4 independent predictors of the presence of CAP after 1 year. The cohort as a whole had a $37.1 \%$ probability of having CAP after 1 year ${ }_{i}$ however, the probability ranged from $19.4 \%$ in children with none of these predictors to $65.5 \%$ among those having 3 or 4 . Adequate follow-up of these children therefore seems warranted.

Nevertheless, these predictors explain only $14.3 \%$ of the variance in the outcome measure, implying that on the basis of simple medical evaluation alone, the family physician is unable to accurately predict the prognosis of a child consulting with abdominal pain. Apparently, other yet to be identified psychosocial, environmental, or family stressors play a role. This complex multifactorial etiology implies that identifying potential prognostic factors in children with a poor prognosis might require a thorough and potentially more time-consuming evaluation of these factors. It also raises the question as to whether children who have persistent pain after adequate follow-up by their family physician should be managed by the family physician alone, or whether one should opt for a primary care multidisciplinary team including health care workers trained in evaluating psychosocial, environmental, and family stressors. 
As long as there is no evidence-based (psychosocial) treatment, however, we believe that a stringent, proactive follow-up of the child with medically unexplained abdominal pain by the family physician is warranted. During follow-up, worsening of the pain or appearance of additional symptoms may alert the family physician to an organic cause or psychosocial problems that become evident after several visits. In such cases, the family physician might request additional diagnostic testing, refer to a secondary care (mental health care) specialist, or treat the child where appropriate.

Given the high level of CAP sufficient to affect well-being among children seen by family physicians, future research is warranted. This research should focus on identifying other risk factors involved in the development and persistence of CAP in primary care.

\section{To read or post commentaries in response to this article, see it} online at http://www.annfammed.org/content/13/2/158.

Key words: abdominal pain; prognosis; gastrointestinal diseases; primary health care; child; practice-based research

Submitted February 11, 2014; submitted, revised, August 5, 2014; accepted September 9, 2014.

Funding support: The study was funded by The Netherlands Organisation for Health Research and Development (ZonMw), grant no. 4200.0008.

Disclaimer: ZonMw did not have any influence on data collection or analysis, or preparation of the manuscript.

Acknowledgments: The authors thank Metthilde Bos, Toke Mulder, Marlies Luiten, Ellen Duijster, and Wilma Bergen Henegouwen for their assistance in data collection and data management. Furthermore, we thank Marieke Gieteling for her contribution in the data-analysis on the $\mathrm{CBCL}$ and somatic syndrome scale.

\section{References}

1. Gieteling MJ, Lisman-van Leeuwen $Y$, van der Wouden JC, Schellevis FG, Berger MY. Childhood nonspecific abdominal pain in family practice: incidence, associated factors, and management. Ann Fam Med. 2011;9(4):337-343.

2. BEACH Program, AIHW General Practice Statistics and Classification Unit. Presentations of abdominal pain in Australian general practice. Aust Fam Physician. 2004;33(12):968-969.

3. Starfield B, Hoekelman RA, McCormick M, et al. Who provides health care to children and adolescents in the United States? Pediatrics. 1984;74(6):991-997.

4. Rasquin A, Di Lorenzo C, Forbes D, et al. Childhood functional gastrointestinal disorders: child/adolescent. Gastroenterology. 2006;130(5): 1527-1537.

5. Chitkara DK, Rawat DJ, Talley NJ. The epidemiology of childhood recurrent abdominal pain in western countries: a systematic review. Am J Gastroenterol. 2005;100(8):1868-1875.

6. Di Lorenzo C, Colletti RB, Lehmann HP, et al; AAP Subcommittee; NASPGHAN Committee on Chronic Abdominal Pain. Chronic Abdominal Pain in Children: a Technical Report of the American Academy of Pediatrics and the North American Society for Pediatric Gastroenterology, Hepatology and Nutrition. J Pediatr Gastroenterol Nutr. 2005;40(3):249-261.

7. Youssef NN, Murphy TG, Langseder AL, Rosh JR. Quality of life for children with functional abdominal pain: a comparison study of patients' and parents' perceptions. Pediatrics. 2006;117(1):54-59.
8. Gieteling MJ, Bierma-Zeinstra SM, Passchier J, Berger MY. Prognosis of chronic or recurrent abdominal pain in children. J Pediatr Gastroenterol Nutr. 2008;47(3):316-326.

9. Howell S, Poulton R, Talley NJ. The natural history of childhood abdominal pain and its association with adult irritable bowel syndrome: birth-cohort study. Am J Gastroenterol. 2005;100(9):2071-2078.

10. Hotopf M, Carr S, Mayou R, Wadsworth M, Wessely S. Why do children have chronic abdominal pain, and what happens to them when they grow up? Population based cohort study. BMJ. 1998;316(7139): 1196-1200.

11. Hyams JS, Treem WR, Justinich CJ, Davis P, Shoup M, Burke G. Characterization of symptoms in children with recurrent abdominal pain: resemblance to irritable bowel syndrome. J Pediatr Gastroenterol Nutr. 1995;20(2):209-214.

12. Chitkara DK, van Tilburg MA, Blois-Martin N, Whitehead WE. Early life risk factors that contribute to irritable bowel syndrome in adults: a systematic review. Am J Gastroenterol. 2008;103(3):765-774, quiz 775.

13. Gieteling MJ, Bierma-Zeinstra SM, Lisman-van Leeuwen Y, Passchier J, Berger MY. Prognostic factors for persistence of chronic abdominal pain in children. J Pediatr Gastroenterol Nutr. 2011;52(2):154-161.

14. Dengler-Crish CM, Horst SN, Walker LS. Somatic complaints in childhood functional abdominal pain are associated with functional gastrointestinal disorders in adolescence and adulthood. J Pediatr Gastroenterol Nutr. 2011;52(2):162-165.

15. Spee LA, van den Hurk AP, van Leeuwen $Y$, et al. Childhood abdominal pain in primary care: design and patient selection of the HONEUR abdominal pain cohort. BMC Fam Pract. 2010;11:27.

16. von Baeyer CL, Walker LS. Children with recurrent abdominal pain: issues in the selection and description of research participants. J Dev Behav Pediatr. 1999;20(5):307-313.

17. Malaty HM, Abudayyeh S, Fraley K, Graham DY, Gilger MA, Hollier DR. Recurrent abdominal pain in school children: effect of obesity and diet. Acta Paediatr. 2007;96(4):572-576.

18. Huang RC, Palmer LJ, Forbes DA. Prevalence and pattern of childhood abdominal pain in an Australian general practice. J Paediatr Child Health. 2000;36(4):349-353.

19. Huntley ED, Campo JV, Dahl RE, Lewin DS. Sleep characteristics of youth with functional abdominal pain and a healthy comparison group. J Pediatr Psychol. 2007;32(8):938-949.

20. Spee LA, Madderom MB, Pijpers M, van Leeuwen Y, Berger MY. Association between Helicobacter pylori and gastrointestinal symptoms in children. Pediatrics. 2010;125(3):e651-e669.

21. Cole TJ, Bellizzi MC, Flegal KM, Dietz WH. Establishing a standard definition for child overweight and obesity worldwide: international survey. BMJ. 2000;320(7244):1240-1243.

22. McGrath PA. Pain in Children: Nature, Assessment, and Treatment. New York, NY: Guilford Press; 1990.

23. Achenbach TM, Rescorla LA. Manual for the ASEBA School-aged Forms and Profiles. Burlington, VT: University of Vermont, Research Center for Children, Youth, \& Families; 2001.

24. Achenbach TM, Rescorla LA. Multicultural Supplement of the Manual for the ASEBA School Age Forms \& Profiles. Burlington, VT: University of Vermont, Research Center for Children, Youth E Families; 2007.

25. Sauerbrei $W$. The use of resampling methods to simplify regression models in medical statistics. J R Stat Soc Ser C Appl Stat. 1999;48(3): 313-329.

26. Perquin CW, Hunfeld JA, Hazebroek-Kampschreur AA, et al. The natural course of chronic benign pain in childhood and adolescence: a twoyear population-based follow-up study. Eur J Pain. 2003;7(6):551-559.

27. Helgeland H, Van Roy B, Sandvik L, Markestad T, Kristensen H. Paediatric functional abdominal pain: significance of child and maternal health. A prospective study. Acta Paediatr. 2011;100(11):1461-1467.

28. Malfertheiner P, Megraud F, O'Morain C, et al. Current concepts in the management of Helicobacter pylori infection: the Maastricht III Consensus Report. Gut. 2007;56(6):772-781.

29. Mulvaney S, Lambert EW, Garber J, Walker LS. Trajectories of symptoms and impairment for pediatric patients with functional abdominal pain: a 5-year longitudinal study. J Am Acad Child Adolesc Psychiatry. 2006;45(6):737-744. 